\title{
Os fundamentos quânticos da Ressonância Magnética Nuclear
}

\author{
Quantum mechanical principles of Nuclear Magnetic Resonance
}

\author{
Celia M. Tokarski Dieguez ${ }^{1}$, Lecio V. Montanheiro ${ }^{1}$, L. Berbeka Cleto ${ }^{1}$, Marlio J.C. Bonfim ${ }^{1}$, C.A. \\ Dartora*1 $^{* 1}$ \\ ${ }^{1}$ Universidade Federal do Paraná, Departamento de Engenharia Elétrica, Curitiba, PR, Brasil
}

Recebido em 22 de Março, 2017. Revisado em 16 de Maio, 2017. Aceito em 20 de Julho, 2017.

\begin{abstract}
A ressonância magnética nuclear (RMN) encontra inúmeras aplicações em Física, Química, Engenharia e Medicina. Ela é utilizada sobretudo na análise de composição e estrutura de materiais, tanto sólidos quanto líquidos, e na realização de imagens tomográficas, na área médica, sem a necessidade de submeter o paciente à qualquer tipo de radiação ionizante. O objetivo do presente trabalho é apresentar para estudantes de Física, Química e Engenharia os conceitos físicos básicos da ressonância magnética nuclear, utilizando a mecânica quântica para deduzir as equações de Bloch e apresentar suas soluções em regime de alto campo magnetostático. As condições necessárias para possibilitar o emprego dessa técnica na prática, utilizando a ressonância do próton, serão discutidas em linhas gerais.
\end{abstract}

Palavras-chave: ressonância magnética nuclear, spin, próton

\begin{abstract}
The phenomenon of nuclear magnetic resonance (NMR) has numerous applications in Physics, Chemistry, Engineering and Medicine, being used for the analysis materials composition and structure, both in solids and liquids. Medical uses are in tomographic imaging, without the need of submitting the patient to ionizing radiation of any kind. The aim of the present manuscript is to introduce the physical principles of NMR using quantum mechanics in order to deduce Bloch's equations and obtain their solutions in the high magnetostatic field regime. The conditions required for practical uses of the technique, using proton's resonance, will be briefly discussed.

Keywords: nuclear magnetic resonance, spin, proton
\end{abstract}

\section{Introdução}

Os estudos com a ressonância magnética nuclear(em inglês NMR - Nuclear Magnetic Resonance) têm suas origens nos anos 1930, através dos trabalhos pioneiros de Isidor Rabi 1 3], R.V. Pound e E.M. Purcell [4] e Felix Bloch [5], culminando no desenvolvimento da espectroscopia NMR por transformada de Fourier [6, 7]. Tal é a importância do assunto que rendeu o prêmio Nobel de Física para I. Rabi em 1944 e para F. Bloch e E.M. Purcell em 1952.

Sabe-se que, em geral, o momento magnético do elétron é o maior responsável pela resposta magnética dos materiais. No entanto, os núcleos atômicos, compostos de prótons e nêutrons, também apresentam momento magnético devido ao spin nuclear resultante. O momento magnético do elétron é usualmente três ordens de grandeza maior do que o momento magnético devido ao spin nuclear de um átomo. Dessa forma, a aplicação de campo magnético externo produz um efeito muito mais pronunciado sobre os elétrons não pareados, que tendem a ficar alinhados anti-paralelamente a esse campo. Campos magnéticos na

*Endereço de correspondência: cadartora@eletrica.ufpr.br ordem de centenas ou até mesmo algumas dezenas de mT podem saturar uma amostra de material no que tange a magnetização resultante produzida pelos elétrons.

Para produzir transições entre o estado magnético fundamental e algum estado excitado do sistema de spins eletrônicos, produzindo o spin-flip de alguns elétrons, é necessário aplicar um campo de radiofrequência(RF) com frequências na faixa das microondas, tipicamente maiores do que dezenas de gigahertz, cujos fótons possuem a escala de energia necessária para produzir a transição, levando o elétron ao estado alinhado ao campo magnetostático. Já os spins nucleares devem ser submetidos a campos muito altos em temperatura ambiente para que ocorra uma magnetização apreciável, mas nesse , caso a ressonância, correspondendo à transição entre o estado alinhado e o anti-alinhado ao campo magnetostático externamente aplicado, é acessível na escala de megahertz. $\mathrm{O}$ valor de momento magnético associado ao spin nuclear depende não somente do tipo de átomo, como também das ligações químicas que este faz, pois o campo magnético produzido pelo alinhamento dos dipolos magnéticos eletrônicos modifica o valor de campo magnético efetivamente percebido pelo núcleo. 
Embora experimentos acessando a ressonância magnética de um elétron, conhecida como EPR ( do inglês - Electron Paramagnetic Resonance), permitam determinar algumas propriedades dos materiais, através do fator $g$ de Landé, a ressonância magnética nuclear é bem mais informativa, tornando possível estudar a composição de um material e o tipo de ligação química que uma determinada espécie química está realizando. Dessa forma, a NMR encontra inúmeras aplicações em Física, Química, Engenharia e Medicina 8 13. Ela é utilizada sobretudo na análise de composição de materiais, tanto sólidos quanto líquidos, bem como na realização de imagens tomográficas na área médica, sem a necessidade de submeter o paciente à qualquer tipo de radiação ionizante. Na Química o uso da NMR rendeu dois prêmios Nobel, para Richard R. Ernst 6, 7] em 1991, pelo desenvolvimento de metodologias de alta resolução na espectroscopia NMR, e para Kurt Wüthrich [14 no ano de 2002, pelo uso da técnica de espectroscopia de NMR na identificação das estruturas tridimensionais de macromoléculas biológicas em solução. Além disso foram lareados com o prêmio Nobel em Fisiologia ou Medicina os pesquisadores Paul C. Lauterbur e Peter Mansfield, no ano de 2003, pelos estudos pioneiros no desenvolvimento da Ressonância Magnética por Imagem (MRI - Magnetic Resonance Imaging) 15 17. O desenvolvimento dos materiais supercondutores cerâmicos de altas temperaturas (maiores que $77 \mathrm{~K}$ ) ainda permitiu a construção de máquinas de tomografia e imagem por NMR utilizando altos campos magnéticos, que chegam até a escala de $10 \mathrm{~T}$.

O objetivo do presente trabalho é apresentar para estudantes de Física, Química e Engenharia os princípios físicos da ressonância magnética nuclear, utilizando a mecânica quântica para deduzir as equações de Bloch, e posteriormente encontrando as suas soluções no regime de alto campo magnetostático aplicado. As condições necessárias para possibilitar o emprego dessa técnica, utilizando a ressonância do próton, serão discutidas de forma geral. Espera-se, como pré-requisito para a compreensão da análise apresentada, que o leitor tenha algum conhecimento de mecânica quântica e da notação de Dirac, ao nível dos três primeiros capítulos do livro-texto de J.J. Sakurai [18].

O conteúdo do presente trabalho está organizado da seguinte maneira: o Hamiltoniano de spin utilizado para deduzir as equações de Bloch será apresentado na Seção 2, que ainda traz a relação entre o campo magnético aplicado e o momento magnético resultante, como função da temperatura; na Seção 3 a solução das equações de Bloch em regime de alto campo magnetostático superposto a um campo de RF transversal será discutida; na Seção 4 as condições gerais para permitir medidas experimentais de NMR serão discutidas em termos de aparatos simples. Finalmente, na Seção 5 são apresentadas as conclusões gerais deste trabalho.

\section{Princípios físicos da Ressonância Magnética Nuclear}

O ponto de partida para a análise da ressonância magnética é o Hamiltoniano descrevendo a interação de Zeeman entre um momento de dipolo magnético e um campo magnético externamente aplicado [11, 18, 19]:

$$
\hat{H}=-\boldsymbol{\mu} \cdot \mathbf{B},
$$

onde $\boldsymbol{\mu}$ é o momento de dipolo magnético da partícula (medido em A.m ${ }^{2}$ no SI) e B é o vetor de indução magnética (medido em $\mathrm{T}$ no SI). O momento de dipolo magnético relaciona-se ao momento angular de uma partícula através de uma constante $\gamma$ denominada razão giromagnética, que tem unidades de $\mathrm{C} / \mathrm{kg}$ no SI. Considerando-se apenas o momento angular de spin, temse:

$$
\boldsymbol{\mu}=\frac{g q}{2 m} \mathbf{S}=\gamma \mathbf{S},
$$

$g$ é denominado fator giromagnético ou simplesmente fator $g$ de Landé, $q$ é a carga elétrica da partícula e $m$ a sua massa, $\mathbf{S}=\left(S_{x}, S_{y}, S_{z}\right)$ é o operador de momento angular da spin. Cabe notar que algumas partículas efetivamente neutras mas dotadas de estrutura interna, como o nêutron, composto de quarks, podem possuir momento de dipolo magnético intrínseco. Na mecânica quântica, o momento angular deve satisfazer as seguintes propriedades:

$$
\begin{aligned}
{\left[S_{i}, S_{j}\right] } & =i \hbar \varepsilon_{i j k} S_{k}, \\
\mathbf{S}^{2}\left|S, m_{z}\right\rangle & =\hbar^{2} S(S+1)\left|S, m_{z}\right\rangle \\
S_{z}\left|S, m_{z}\right\rangle & =\hbar m_{z}\left|S, m_{z}\right\rangle
\end{aligned}
$$

sendo $[A, B]=A B-B A$ o comutador entre os operadores $A$ e $B, S_{i}$ é a componente $i$-ésima do vetor $\mathbf{S}$ e $\hbar=$ $1,055 \times 10^{-34}$ J.s é a constante de Planck. O número de spin $S$ pode assumir apenas valores inteiros ou semiinteiros $(S=0,1 / 2,1,3 / 2 \ldots)$. Para um dado valor de $S$ a projeção do momento angular de spin na direção de $z$ pode somente assumir os valores $m_{z}=-S,-S+1, \ldots S-$ $1, S$. Dessa forma, os ket-estados $\left|S, m_{z}\right\rangle$ no espaço de Hilbert descrevem os autoestados possíveis do operador $S_{z}$ e simultaneamente de $\mathbf{S}^{2}$. Para o caso do spin $S=$ $1 / 2$, que consideramos mais relevante para o presente trabalho, temos $m_{z}=-1 / 2,+1 / 2$, correspondendo às orientações anti-paralela e paralela em relação a um campo magnético orientado ao longo do eixo $z$. O tensor de Levi-Civitta, denotado por $\varepsilon_{i j k}$, é anti-simétrico pela permuta de índices $i j k$ e assume os valores +1 para $i j k=\{x y z, y z x, z x y\},-1$ para $i j k=\{y x z, z y x, x z y\}$ ou 0 para o caso em que qualquer índice apareça repetido.

Para a obtenção das equações de movimento podemos aplicar a versão de Heisenberg da mecânica quântica, onde um operador $\hat{O}$ qualquer deve satisfazer à seguinte equação 18]:

$$
i \hbar \frac{d \hat{O}}{d t}=[\hat{O}, \hat{H}]
$$


Fazendo $\hat{O}=\boldsymbol{\mu}=\gamma \mathbf{S}$ na equação acima e tendo em conta o Hamiltoniano (1) e as relações de comutação mostradas em (3), fica como exercício para o leitor interessado demonstrar que o operador de momento de dipolo magnético satisfaz à seguinte equação:

$$
\frac{d \boldsymbol{\mu}}{d t}=\gamma \boldsymbol{\mu} \times \mathbf{B}
$$

É importante destacar que esse conjunto de equações, condensado na forma de uma única equação diferencial vetorial, é fundamental para compreender a NMR, sendo conhecido como equações de Bloch. Na forma apresentada, as equações de Bloch são originadas tão somente do acoplamento de Zeeman entre o momento magnético e o campo magnético aplicado, não levando em conta efeitos dissipativos, sempre presentes nas situações reais. É possível introduzir dissipação através de termos fenomenológicos da forma $-\mu_{i} / \tau_{i}$, sendo $\tau_{i}$ o tempo de relaxação característico na direção do eixo $i$-ésimo, uma vez que geralmente o tempo de relaxação não é isotrópico [11,12]. Adicionalmente, em materiais ferromagnéticos deve-se introduzir nas equações de Bloch o efeito da interação spin-spin, que leva a uma pronunciada modificação do campo magnético percebido internamente. A situação envolvendo ferromagnetismo foge ao escopo do presente trabalho e por esse motivo não será analisada.

\subsection{Algumas considerações estatísticas}

O leitor pode observar a equação (7) tem forma idêntica ao resultado da mecânica clássica, derivado da análise do torque exercido por forças de origem magnética sobre um momento de dipolo magnético clássico. Ressaltese, entretanto, que (7) é uma equação para o operador mecânico-quântico $\boldsymbol{\mu}=\gamma \mathbf{S}$. Com a finalidade de uma versão clássica a partir do resultado mecânico-quântico, devemos calcular a média da equação (7), utilizando o formalismo da matriz densidade $\hat{\rho}$, que é a correspondente quântica da função de distribuição de probabilidades clássica. Tipicamente, em medidas de NMR os spins estão sujeitos a um campo magnetostático muito intenso, superposto a um campo de RF de menor magnitude, que é utilizado para sondar o sistema. O campo magnético total pode ser expresso na forma $\mathbf{B}=\mathbf{b}(t)+B_{0} \hat{z}$, onde $B_{0}$ é a magnitude do campo magnetostático ao longo do eixo $z$ e $\mathbf{b}(t)$ corresponde ao campo de RF, satisfazendo a condição $|\mathbf{b}(t)|<<B_{0}$. Quando $\mathbf{b}(t)=0$ para $t<0$, ou seja, antes de o campo de RF ser aplicado, podemos considerar o sistema inicialmente em equilíbrio térmico, na presença do campo magnetostático $B_{0} \hat{z}$ somente. A matriz densidade no ensemble canônico para o sistema no equilíbrio é mostrada abaixo:

$$
\hat{\rho}_{0}=\frac{1}{Z} e^{-\beta \hat{H}_{0}}=\frac{1}{Z} \sum_{m_{z}=-S}^{S} e^{-\beta \gamma \hbar m_{z} B_{0}}\left|S, m_{z}\right\rangle\left\langle S, m_{z}\right|,
$$

onde $\hat{H}_{0}=-\gamma B_{0} S_{z}$ corresponde ao termo de Zeeman devido à parcela magnetostática apenas, $\beta=1 /\left(k_{B} T\right)$ é o recíproco da temperatura $T$ (medida em $\mathrm{K}$ ), $k_{B}=1,38 \times$ $10^{-23} \mathrm{~J} / \mathrm{K}$ é a constante de Boltzmann e $Z=\operatorname{tr}\left(e^{-\beta \hat{H}_{0}}\right)$ é a função de partição ( tr denota o traço de matriz) tal que a condição $\operatorname{tr}\left(\hat{\rho}_{0}\right)=1$ seja satisfeita. No presente caso tem-se que:

$$
Z=\sum_{m_{z}=-S}^{S} e^{-\beta \gamma \hbar m_{z} B_{0}}
$$

No contexto do formalismo da matriz densidade, o valor médio estatístico de um operador mecânico-quântico $\hat{O}$ é dado por $\langle\hat{O}\rangle=\operatorname{tr}\left(\hat{\rho}_{0} \hat{O}\right)[18$. Aqui devemos calcular o valor médio da densidade volumétrica de magnetização [20], ou simplesmente vetor magnetização $\mathbf{M}$, definido como o momento de dipolo magnético total contido em um volume $V$ de material, dividido por esse mesmo volume, ou seja, $\mathbf{M}=\left(\sum_{j=1}^{N}\left\langle\mu_{j}\right\rangle\right) / V$, medido em A/m no SI, na forma abaixo:

$$
\mathbf{M}=n\langle\boldsymbol{\mu}\rangle=n \operatorname{tr}\left(\hat{\rho}_{0} \boldsymbol{\mu}\right) .
$$

O resultado acima é válido quando assumimos que que no interior do volume $V$ o valor médio de todos os momentos individuais $\mu_{j}$ é idêntico aos demais, e portanto temos $N$ momentos idênticos em um volume $V$, o que resulta simplesmente na densidade de momentos de dipolo magnético do material denotada por $n=N / V$. Desse modo, podemos tomar a média da equação (7) e multiplicá-la por $n$ para obter a equação de movimento clássica, dada na forma abaixo:

$$
\frac{d \mathbf{M}}{d t}=\gamma \mathbf{M} \times \mathbf{B}
$$

Agora podemos calcular a condição inicial do sistema, ou seja, a magnetização efetiva para $t<0$, um pouco antes de o campo de RF ser ligado, uma vez que o campo magnetostático orienta os momentos magnéticos nucleares, produzindo assim uma magnetização efetiva do sistema, que está em equilíbrio térmico. Nesse caso, a condição inicial para determinação da dinâmica da magnetização em função do tempo toma a forma $\mathbf{M}(t=$ $0)=\left(0,0, M_{0}\right)$, sendo

$$
M_{0}=-n \frac{\partial}{\partial\left(\beta B_{0}\right)} \ln (Z)=-n \gamma \hbar \frac{\sum_{m_{z}=-S}^{S} m_{z} e^{-\beta \gamma \hbar m_{z} B_{0}}}{\sum_{m_{z}=-S}^{S} e^{-\beta \gamma \hbar m_{z} B_{0}}} .
$$

O caso de maior interesse é o de spin $S=1 / 2$, quando a função acima reduz-se à seguinte expressão:

$$
M_{0}=n \mu_{p} \tanh \left(\beta \mu_{p} B_{0}\right),
$$

onde definiu-se a quantidade $\mu_{p}=\gamma \hbar / 2$, denominado momento magnético intrínseco da partícula, ou magnéton, medido em J/T. Para um elétron o valor dessa quantidade é de aproximadamente um magnéton de Bohr, $\mu_{B}=9,274009 \times 10^{-24} \mathrm{~J} / \mathrm{T}$, sem levar em conta as correções provenientes da eletrodinâmica quântica, enquanto que para um próton relaciona-se ao chamado 
magnéton nuclear $\mu_{N}=5,050783 \times 10^{-27} \mathrm{~J} / \mathrm{T}$, na forma $\mu_{p}=2,792847 \mu_{N}=1,410606 \times 10^{-26} \mathrm{~J} / \mathrm{T}$. Como mencionado anteriormente, o momento magnético de um elétron é 3 ordens de grandeza maior que o do próton. Uma vez que para o próton tipicamente temos $\mu_{p} B_{0}<<k_{B} T$, podemos fazer $\tanh (x) \approx x$ na equação 13 , o que nos fornece o seguinte resultado:

$$
M_{0}=n \mu_{p} \frac{\mu_{p} B_{0}}{k_{B} T} .
$$

A quantidade $\frac{\mu_{p} B_{0}}{k_{B} T}$ na expressão acima corresponde à diferença entre o número de spins alinhados e o número de spins anti-alinhados ao campo $B_{0}$, normalizada pelo número total de partículas. No caso em que $T=300 \mathrm{~K}$ e $B_{0}=3$ teslas temos $\frac{\mu_{p} B_{0}}{k_{B} T}=10 \times 10^{-6}$, significando que para um número total de $10^{6}$ spins, há apenas 10 spins alinhados a mais do que anti-alinhados ao campo magnetostático. Todavia precisamos saber como calcular o valor de $M_{0}$ para um determinado material, que depende ainda da densidade de momentos de dipolo, $n$. Primeiro é preciso especificar qual é o átomo de interesse no estudo, o que define o valor de $\mu_{p}$ bem como a densidade de partículas $n$ em função da sua proporção no material. É importante mencionar que uma parte considerável dos núcleos atômicos não responde de forma significativa ao campo magnético através de momento de dipolo magnético, porque seu estado fundamental tem spin total zero. Isso ocorre para os núcleos contendo número par de prótons e de nêutrons em seu interior, como é o caso do carbono 12, que tem 6 prótons e 6 nêutrons. No entanto, isótopos com número de massa ímpar irão apresentar pelo menos um spin que não pode ser pareado e, portanto, respondem ao campo magnético, como é o caso do carbono 13. O deutério, isótopo do Hidrogênio contendo um próton e um nêutron, irá responder ao campo porque o momento magnético do próton é ligeiramente maior do que o do nêutron.

Uma fórmula simples para determinar a densidade de momentos de dipolo magnético presentes no material é a seguinte:

$$
n=N \frac{\rho_{m}}{m_{u}},
$$

onde $\rho_{m}$ é a densidade de massa do material, $m_{u}$ é a massa de uma unidade fundamental do material (átomo ou molécula) e $N$ é o número de átomos (que respondem ao campo magnético e cuja ressonância se quer determinar) presentes na unidade fundamental do material. O elemento mais comumente empregado no estudo da ressonância nuclear é átomo de hidrogênio $H^{1}$, para o qual $\mu_{p}$ é o momento de dipolo magnético do próton, e $n$ será a densidade de átomos de hidrogênio presentes por unidade de volume do material considerado. Por exemplo, para a água cada molécula $\left(\mathrm{H}_{2} \mathrm{O}\right)$ possui 2 átomos de hidrogênio e é sabido que o isótopo de oxigênio $O^{16}$, mais comum na natureza, não responde ao campo magnético. A densidade da água é de aproximadamente $1000 \mathrm{~kg} / \mathrm{m}^{3}$ e cada molécula de água tem massa total $18 m_{p}$, onde $m_{p}=1,673 \times 10^{-27} \mathrm{~kg}$ é a massa do próton. Dessa forma:

$$
n=2 \frac{1000 \mathrm{~kg} / \mathrm{m}^{3}}{18 m_{p}}=6,6 \times 10^{28} \mathrm{spins} / \mathrm{m}^{3} .
$$

Considerando-se um campo $B_{0}=3 \mathrm{~T}$ aplicado à água em temperatura ambiente $(300 \mathrm{~K})$ teremos $6,6 \times 10^{23}$ prótons alinhados ao campo, o que produz uma densidade de momento de dipolo magnético resultante de valor $M_{0}=9,3$ $\mathrm{mA} / \mathrm{m}$. Fazendo um cálculo rudimentar, isso corresponde, em unidades de indução magnética, a $\mu_{0} M_{0} \approx 11 \mathrm{nT}$, onde $\mu_{0}=4 \pi \times 10^{-7} \mathrm{H} / \mathrm{m}$ é a permeabilidade magnética do vácuo.

Do ponto de vista da mecânica quântica, a ressonância ocorre quando um fóton incidente no núcleo tem energia igual à diferença de energia entre dois níveis energéticos consecutivos do sistema de spins. Quanto o fóton é absorvido produz uma transição entre esse níveis. No caso de spin 1/2, o fóton é capaz de fazer o spin girar do estado alinhado para o estado anti-alinhado. Considerando-se o Hamiltoniano $\hat{H}_{0}=-\gamma S_{z} B_{0}$ no campo magnetostático $B_{0}$, o estado de spin para cima (alinhado) tem menor energia, $-\mu_{p} B_{0}$, enquanto que o estado de spin para baixo (anti-alinhado) tem maior energia, $+\mu_{p} B_{0}$. A relação entre a energia do fóton e a diferença de energia entre esses dois níveis é dada por:

$$
\hbar \omega_{0}=\Delta E=2 \mu_{p} B_{0}
$$

onde $\omega_{0}$ é a frequência angular do fóton (medida em $\mathrm{rad} / \mathrm{s}$ ), denominada de frequência de Larmor do sistema, pois corresponde ao valor clássico de frequência de precessão de spin. Para o próton em um campo $B_{0}=1 \mathrm{~T}$ temos que $f_{0}=\omega_{0} /(2 \pi) \approx 42,577 \mathrm{MHz}$.

Para encerrar essa Seção, cabe mencionar que os tempos de relaxação $\tau$ em problemas de NMR típicos, envolvendo líquidos ou sólidos paramagnéticos ou diamagnéticos, são maiores que $1 \mathrm{~ms}$, podendo chegar à escala de segundos. Se estamos interessados em analisar aspectos dinâmicos em escalas de tempo de até $100 \mu$ s é plausível negligenciar os efeitos dissipativos, em primeira aproximação. Por fins de simplicidade vamos desconsiderar os efeitos de dissipação no que segue.

\section{Solução das Equações de Bloch}

Com o intuito de sondar o sistema através da técnica de NMR se faz necessária a aplicação de um campo de RF. As equações de Bloch (11) na presença do campo magnético total, $\mathbf{B}=\mathbf{b}(t)+B_{0} \hat{z}$, onde o campo de $\mathrm{RF} \mathbf{b}(t)$ é ligado para $t>0$, são mostradas abaixo, explicitamente, em forma de componentes:

$$
\begin{aligned}
\frac{d M_{x}}{d t} & =\gamma\left(M_{y} B_{z}-M_{z} B_{y}\right) \\
\frac{d M_{y}}{d t} & =\gamma\left(M_{z} B_{x}-M_{x} B_{z}\right) \\
\frac{d M_{z}}{d t} & =\gamma\left(M_{x} B_{y}-M_{y} B_{x}\right) .
\end{aligned}
$$


Tipicamente, na técnica de NMR em alto campo magnetostático o valor deste é pelo menos uma ordem de grandeza maior do que a amplitude do campo de RF aplicado ao sistema. Vamos supor que o campo magnético total seja dado na forma $\mathbf{B}=b_{0} \cos (\omega t) \hat{x}+B_{0} \hat{z}$, onde $B_{0}$ é o campo magnetostático, $b_{0}<<B_{0}$ é a amplitude do campo de RF e $\omega$ é a sua frequência angular temporal, conhecida como frequência portadora ou carreadora. Note que $B_{y}=0$, sem perda de generalidade. Desse modo, utilizando o fato de que $\cos (\omega t)=\left(e^{i \omega t}+e^{-i \omega t}\right) / 2$, as equações tomam a forma abaixo:

$$
\begin{aligned}
\frac{d M_{x}}{d t} & =\omega_{0} M_{y} \\
\frac{d M_{y}}{d t} & =\frac{\gamma b_{0}}{2}\left(e^{i \omega t}+e^{-i \omega t}\right) M_{z}-\omega_{0} M_{x} \\
\frac{d M_{z}}{d t} & =-\frac{\gamma b_{0}}{2}\left(e^{i \omega t}+e^{-i \omega t}\right) M_{y}
\end{aligned}
$$

onde $\omega_{0}=\gamma B_{0}$ é a frequência de Larmor. O sistema de equações acima não é tão simples de ser resolvido porque, embora seja linear, os coeficientes dependem do tempo. O artifício para permitir a solução é transformar o sistema para um referencial de eixos girantes que acompanham a precessão da magnetização no plano $(x, y)$, com frequência angular de giro igual a frequência angular de Larmor $\omega_{0}$, fazendo $M_{x}(t)=m_{x}(t) e^{i \omega_{0} t}$ e $M_{y}(t)=m_{y}(t) e^{i \omega_{0} t}$. Substituindo essas expressões no sistema de equações e negligenciando $d m_{x} / d t$ em (21), podemos concluir que $m_{x}(t)=-i m_{y}(t)$. Consequentemente, o sistema a ser resolvido reduz-se a duas equações acopladas, mostradas abaixo:

$$
\begin{aligned}
\frac{d m_{y}}{d t} & =\frac{\gamma b_{0}}{2}\left(e^{i\left(\omega-\omega_{0}\right) t}-e^{-i\left(\omega+\omega_{0}\right) t}\right) M_{z} \\
\frac{d M_{z}}{d t} & =-\frac{\gamma b_{0}}{2}\left(e^{i\left(\omega+\omega_{0}\right) t}+e^{-i\left(\omega-\omega_{0}\right) t}\right) m_{y} .
\end{aligned}
$$

Na aproximação de onda girante (RWA - do inglês Rotating Wave Approximation) [21] ou ainda denominada aproximação secular, termos da forma $\omega+\omega_{0}$ tem variações muito rápidas e são negligenciáveis, pois produzem contribuição desprezível quando as equações acima são integradas. Desse modo, vamos reter somente os termos que dependem da diferença de frequência angular $\Delta \omega=\omega-\omega_{0}$, conhecida no jargão da Química como offset, e considerar soluções na forma $m_{y}(t)=A_{y} e^{i \omega_{y} t} \mathrm{e}$ $M_{z}(t)=A_{z} e^{i \omega_{z} t}$. A condição de casamento de fase implica que $\omega_{y}=\Delta \omega+\omega_{z}$, como pode ser demonstrado pelo leitor. Adicionalmente, o sistema se reduz a encontrar os autovalores e autovetores da seguinte equação:

$$
\left(\begin{array}{cc}
i \omega_{y} & -\frac{\gamma b_{0}}{2} \\
\frac{\gamma b_{0}}{2} & i\left(\omega_{y}-\Delta \omega\right)
\end{array}\right)\left(\begin{array}{c}
A_{y} \\
A_{z}
\end{array}\right)=0
$$

O cálculo dos autovalores, fazendo-se o determinante da matriz acima igual a zero, nos fornece o seguinte resultado:

$$
\begin{gathered}
\omega_{y}^{ \pm}=\frac{\Delta \omega}{2} \pm \frac{1}{2} \sqrt{(\Delta \omega)^{2}+\left(\gamma b_{0}\right)^{2}}, \\
\omega_{z}^{ \pm}=-\frac{\Delta \omega}{2} \pm \frac{1}{2} \sqrt{(\Delta \omega)^{2}+\left(\gamma b_{0}\right)^{2}} .
\end{gathered}
$$

Conforme podemos ver, há dois autovalores distintos para $\omega_{y}$ e $\omega_{z}$, e consequentemente dois autovetores distintos. A relação entre as amplitudes $A_{y}$ e $A_{z}$, que são as componentes dos autovetores do sistema, está dada na forma que segue:

$$
A_{y}^{ \pm}=-i \frac{\gamma b_{0}}{2 \omega_{y}^{ \pm}} A_{z}^{ \pm}
$$

A solução mais geral possível para o sistema de equações de Bloch na aproximação RWA é mostrada abaixo:

$$
\begin{aligned}
& m_{y}(t)=A_{y}^{+} e^{i \omega_{y}^{+} t}+A_{y}^{-} e^{i \omega_{y}^{-} t}, \\
& M_{z}(t)=A_{z}^{+} e^{i \omega_{z}^{+} t}+A_{z}^{-} e^{i \omega_{z}^{-} t} .
\end{aligned}
$$

Agora podemos impor a condição inicial sobre $M_{z}(t)$, pois sabemos que $M_{z}(0)=M_{0}$, cujo valor é induzido pelo campo magnetostático, conforme discutido na Seção anterior. Essa condição implica que $A_{z}^{+}+A_{z}^{-}=M_{0}$. Admitindo-se que $A_{z}^{+}=A_{z}^{-}=M_{0} / 2$ obtemos uma solução automaticamente real para $M_{z}(t)$. Fica como exercício para o leitor demonstrar que, considerando-se a parte real de $m_{y}(t) e^{i \omega_{0} t}$ e na condição inicial apresentada, a solução completa do sistema de equações tem a forma abaixo:

$$
\begin{aligned}
M_{y}(t) & =\frac{M_{0}}{\gamma b_{0}}\left[\Delta \omega \cos (\Omega t) \sin \left[\left(\omega_{0}+\frac{\Delta \omega}{2}\right) t\right]\right. \\
& \left.-2 \Omega \sin (\Omega t) \cos \left[\left(\omega_{0}+\frac{\Delta \omega}{2}\right) t\right]\right] \\
M_{z}(t) & =M_{0} \cos \left(\frac{\Delta \omega}{2} t\right) \cos (\Omega t)
\end{aligned}
$$

onde $\Omega=\frac{1}{2} \sqrt{(\Delta \omega)^{2}+\left(\gamma b_{0}\right)^{2}}$ está relacionada à precessão da magnetização em torno do campo de RF. A determinação da componente $M_{x}(t)$ é feita integrando-se a equação 21, dada a função para $M_{y}(t)$ acima.

Na condição de ressonância magnética, o offset anulase, ou seja, $\Delta \omega=\omega-\omega_{0}=0$, e as equações acima reduzem-se às seguintes:

$$
\begin{aligned}
& M_{y}(t)=M_{0} \sin (\Omega t) \cos \left(\omega_{0} t\right), \\
& M_{z}(t)=M_{0} \cos (\Omega t),
\end{aligned}
$$

onde $\Omega=\left(\gamma b_{0}\right) / 2$, nesse caso. Para um campo de RF de amplitude $b_{0}=1 \mathrm{mT}$ e para um próton, cujo valor da constante giromagnética vale $\gamma=2,6752222 \times 10^{8}$ $\mathrm{rad} / \mathrm{s} / \mathrm{T}$, temos $\Omega /(2 \pi) \approx 21,3 \mathrm{kHz}$. Para efeito de comparação, a frequência de ressonância em $B_{0}=1 \mathrm{~T}$ resulta em $f_{0}=\omega_{0} /(2 \pi)=42,577 \mathrm{MHz}$. Portanto a função $M_{z}(t)$ oscila com uma frequência característica $\Omega$ que é nesse caso 3 ordens de magnitude menor do que a 
frequência com que as componentes $M_{y}(t)$ e $M_{x}(t)$ oscilam. Claramente a razão entre $\Omega$ e $\omega_{0}$ na ressonância é dada por $b_{0} /\left(2 B_{0}\right)$. A rotação das componentes $M_{x}$ e $M_{y}$ é ainda modulada por um envelope de frequência angular $\Omega$, que é igual à frequência angular com que $M_{z}(t)$ varia no tempo.

O leitor deve observar que na ressonância a amplitude máxima das funções de $M_{x}$ e $M_{y}$ é igual à magnetização $M_{0}$, obtida através da aplicação do campo magnetostático ao longo do eixo $z$, e não depende essencialmente do valor de amplitude do campo de RF. O que acontece nesse caso é que, quando o sistema é levado para a condição de ressonância, os fótons do campo de RF tem energia suficiente para girar o spin, levando-o ao estado de maior energia. O valor de amplitude do campo de RF, $b_{0}$, está relacionado à taxa de fótons que estão sendo injetados no sistema. Nesse caso, para que ocorra a inversão total das populações de spin, levando a magnetização do sistema de $M_{z}=M_{0}$ para $M_{z}=-M_{0}$, é necessário um intervalo de tempo $t_{i}$ que deve satisfazer $\Omega t_{i}=\pi$, conforme é evidente da equação (35). Isso nos dá um tempo $t_{i}=2 \pi /\left(\gamma b_{0}\right)$, sendo inversamente proporcional à amplitude do campo de RF.

Para fins ilustrativos, o sistema de equações acima foi resolvido numericamente, utilizando uma temperatura de $300 \mathrm{~K}$ em um campo $B_{0}=1 \mathrm{~T}$, o que produz uma frequência de ressonância de aproximadamente $f_{0}=$ 42, $577 \mathrm{MHz}$. Os parâmetros do campo de RF foram $b_{0}=1 \mathrm{mT}$ e $f=\omega /(2 \pi)=f_{0}$, ou seja o sistema foi colocado na ressonância. A Figura 1 ilustra os resultados até um ciclo completo da componente $M_{z}(t)$. Conforme podemos verificar, o período de um ciclo completo para a componente $M_{z}(t)$, quando esta retorna para o seu valor inicial $M_{0} \approx 3 \mathrm{~mA} / \mathrm{m}$ em temperatura ambiente, é aproximadamente $47 \mu \mathrm{s}$, que corresponde a $2 \pi / \Omega$, como é esperado da equação (35). Podemos observar também que a envoltória $\sin (\Omega t)$ está presente na função $M_{y}(t)$, que oscila em uma frequência muito maior do que $\Omega$, conforme esperado.

Já na Figura 2 consideramos a situação em que a frequência do campo de RF está ligeiramente fora da ressonância, com todos os outros parâmetros físicos mantidos idênticos ao da situação ilustrada na Figura 1. O valor escolhido foi $\omega=0,998 \omega_{0}$ e nesse caso é fácil verificar que a componente $M_{z}$ não sofre mais inversão, oscilando próximo do valor inicial $M_{0}$ com uma frequência $\Omega$ que corresponde a 4 vezes o valor do caso ressonante, o que se deve essencialmente à presença de offset $\Delta \omega$. Além disso, $M_{y}(t)$ agora oscila com amplitude menor, sendo próximo de metade da amplitude do caso ressonante. É importante ressaltar que aqui estamos desconsiderando os efeitos de dissipação e, por esse motivo, o sistema é bastante sensível ao offset $\Delta \omega$, tendo uma largura de linha de ressonância bastante estreita. Todavia, a inclusão dos efeitos dissipativos, que contribui para o alargamento da linha de ressonância, não afeta a sensibilidade dos experimentos de NMR, onde offsets na ordem de partes por milhão (ppm) podem ser detectados. Uma vez que, conforme mencionado, o campo magnetostático efetivamente experimentado pelo próton do átomo de hidrogênio (ou pelo núcleo que está sendo considerado para realizar a NMR) depende da ligação química que ele faz, devido ao campo magnético local produzido pelos elétrons de elementos vizinhos, ocorrerá um offset que pode ser detectado experimentalmente, sendo característico da espécie química em análise.

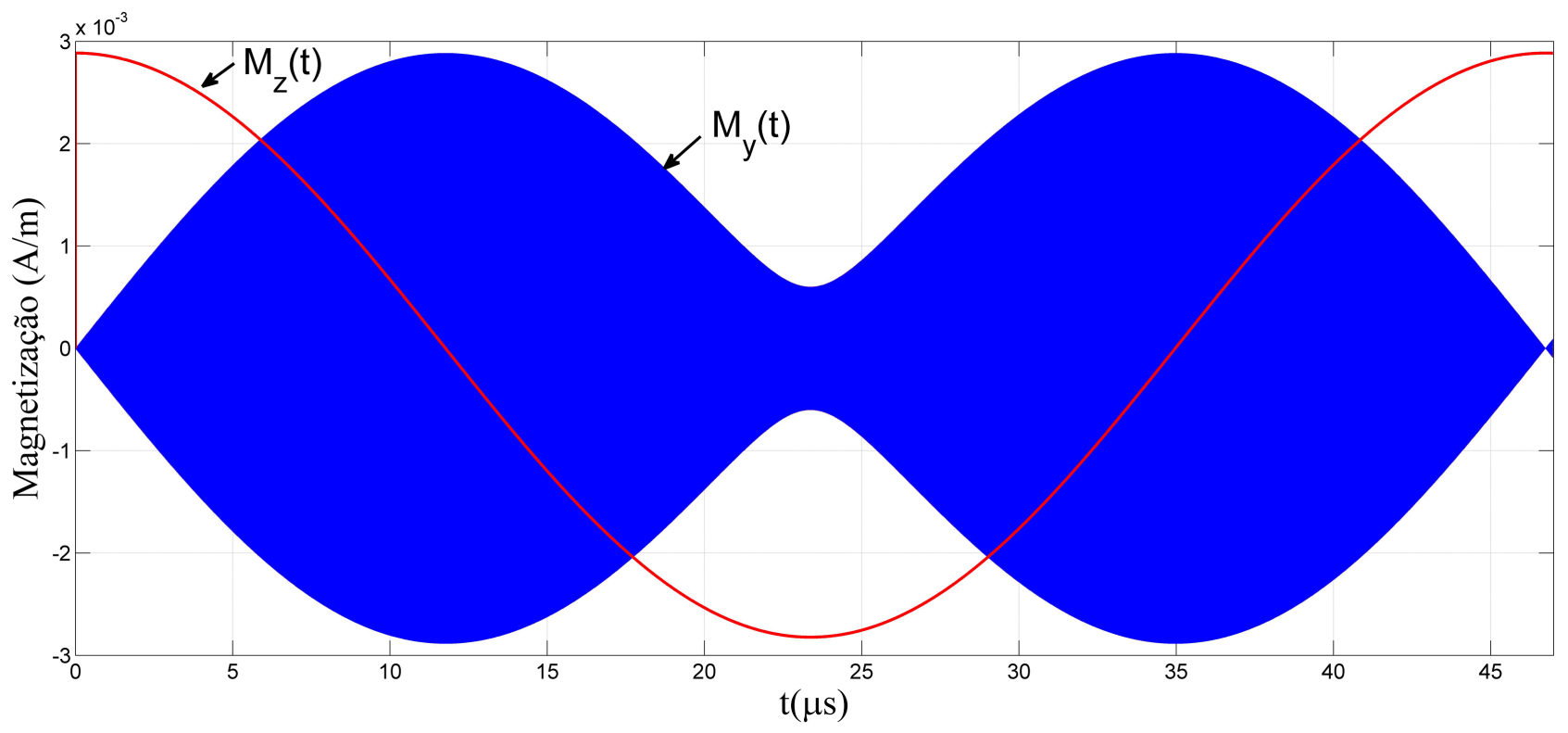

Figura 1: Componentes $M_{y}(t)$ e $M_{z}(t)$ da magnetização da água submetida a um campo magnetostático de amplitude $B_{0}=1$ $\mathrm{T}$ em temperatura ambiente em um experimento típico de NMR. O campo de RF de amplitude $1 \mathrm{mT}$ foi escolhido para oscilar exatamente na frequência de ressonância. 


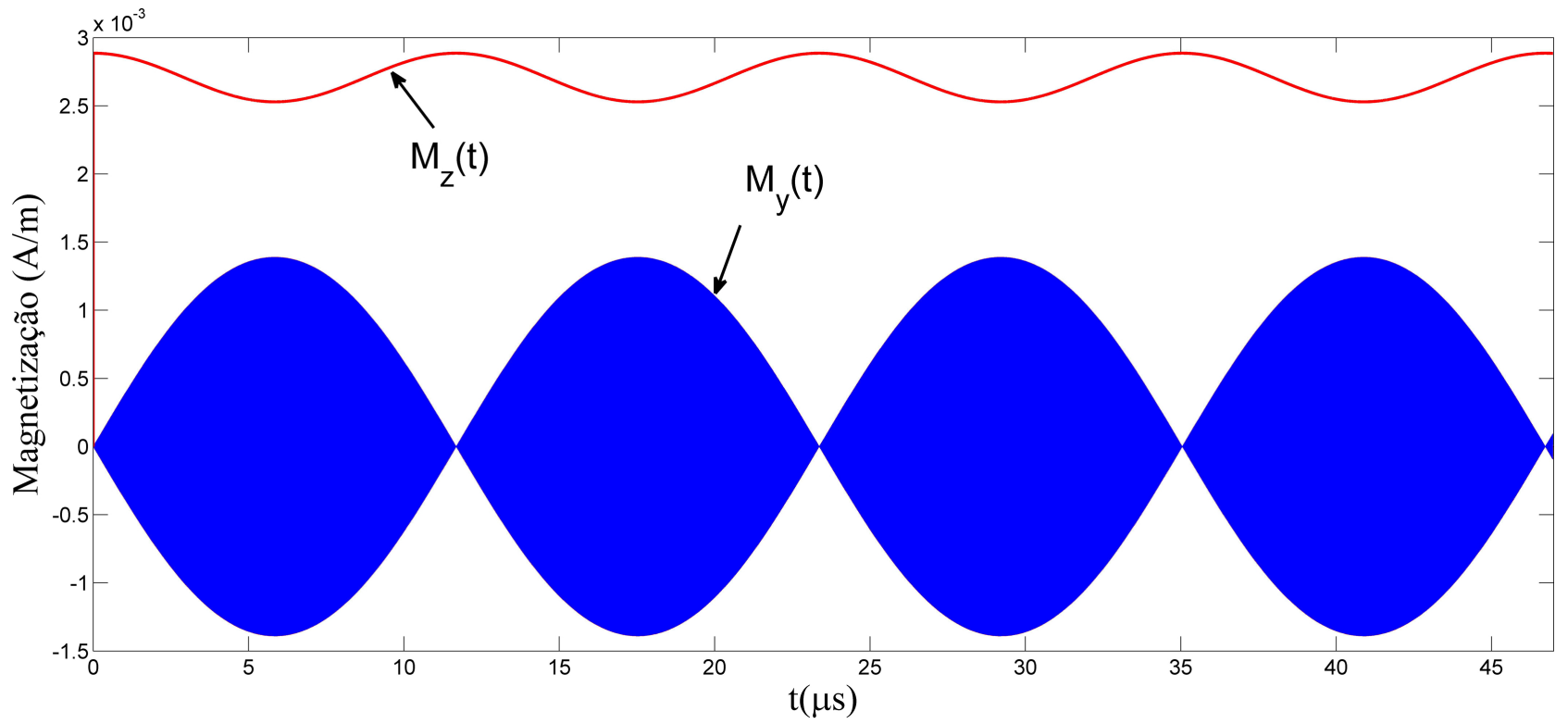

Figura 2: Componentes $M_{y}(t)$ e $M_{z}(t)$ da magnetização da água submetida a um campo magnetostático de amplitude $B_{0}=1 \mathrm{~T}$ em temperatura ambiente em um experimento típico de NMR com a frequência do campo de RF fora da ressonância, amplitude 1 $\mathrm{mT}$ e $\omega=0,998 \omega_{0}$.

O parâmetro $\delta$, denominado deslocamento químico e medido em ppm, define o desvio de frequência de ressonância em relação ao próton isolado (no caso de se utilizar o núcleo do hidrogênio), na forma dada abaixo:

$$
\delta=\frac{\omega_{0}^{\prime}-\omega_{0}}{\omega_{0}} \times 10^{6}[\mathrm{ppm}],
$$

onde $\omega_{0}^{\prime}$ é a ressonância medida no experimento e $\omega_{0}$ a frequência de ressonância de referência no experimento. O deslocamento químico observado nos experimentos está associado às espécies químicas com as quais o hidrogênio está ligado, bem como ao tipo de ligação química realizada, e esta medida é o que torna a técnica de NMR útil na determinação da estrutura da matéria.

\section{Considerações sobre Aparato Experimental}

Um aparato experimental típico de medidas com NMR é ilustrado na Figura 3. Um campo magnetostático, de intensidade $B_{0}$, aplicado ao longo do eixo $z$, é produzido por um eletroímã supercondutor ou um ímã permanente de alto campo. A amostra que se quer caracterizar é imersa nessas linhas de campo. Existem duas bobinas que são ortogonais entre si, para que seus campos magnéticos sejam ortogonais entre si e adicionalmente também ao campo magnetostático. Uma dessas bobinas é utilizada para aplicar o campo de RF e a outra para medir a tensão induzida pela precessão da magnetização da amostra. A tensão medida nos terminais dessa última bobina é levada a um equipamento analisador de sinais. A análise pode ser feita no domínio da frequência, através do cálculo da transformada de Fourier dos sinais medidos no domínio do tempo. Em sistemas de NMR pulsados, uma das bobinas de $\mathrm{RF}$ pode ser dispensada, uma vez que a mesma bobina pode ser utilizada para excitar o sistema através do RF, durante uma janela temporal, e para realizar a medida da rotação da magnetização, em outra janela de tempo, quando o campo de RF encontra-se desligado.

Uma vez que o módulo da magnetização, $M_{0}$, está na ordem de grandeza de $1 \mathrm{~mA} / \mathrm{m}$ em temperatura ambiente, e no plano transversal ao campo magnetostático aplicado as componentes $M_{x}$ e $M_{y}$ oscilam com a frequência de Larmor $\omega_{0}$ quando a condição de ressonânca é satisfeita, podemos determinar a ordem de grandeza das tensões induzidas na bobina de medida. O campo de RF é aplicado a uma das bobinas e a resposta da magnetização é medida no eixo ortogonal, para o caso do uso de duas bobinas cruzadas e independentes. Dada a situação considerada na Seção anterior, quando consideramos que o

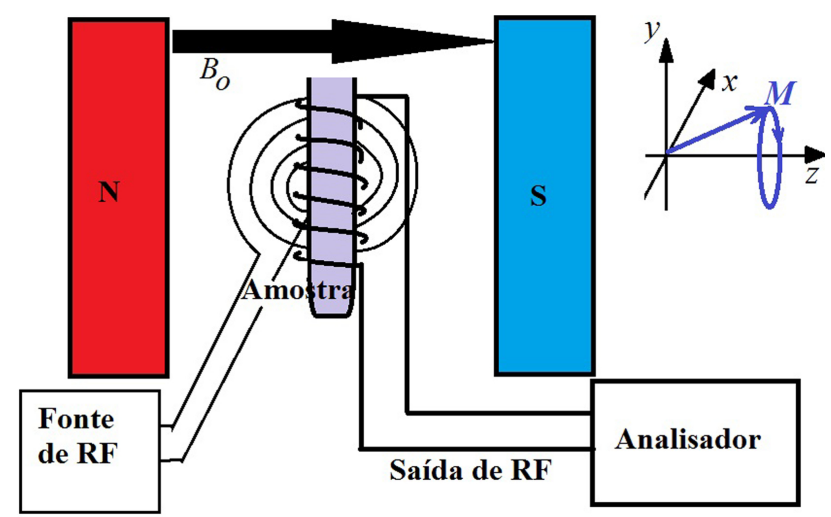

Figura 3: Aparato experimental típico utilizado em um experimento de NMR. 
campo de RF foi aplicado ao longo do eixo $x$, devemos medir a tensão induzida pela componente $M_{y}$ em uma bobina de detecção que é ortogonal àquela da aplicação do campo. Utilizando a lei de Faraday temos:

$$
V_{i n d}=-N_{e} \frac{d \Phi_{m}}{d t}=-N_{e} \frac{d}{d t} \int_{A} \mathbf{B} \cdot d \mathbf{a},
$$

onde $V_{\text {ind }}$ é a tensão induzida na bobina de detecção, $N_{e}$ é o número de voltas da bobina de detecção e $d \mathbf{a}=d x d z \hat{y}$ é o diferencial de área, que deve selecionar a componente $y$ do campo magnético total, uma vez que $B_{y} \approx \mu_{0} M_{y}(t)$, $A$ é a área transversal total da bobina. Se admitimos que o campo magnético produzido pela amostra é uniforme no interior da bobina, e sabendo que o valor máximo de $d M_{y} / d t$ deve valer $\omega_{0} M_{0}$, temos:

$$
\left|V_{i n d}\right|=\mu_{0} N_{e} A \omega_{0} M_{0}
$$

Para uma bobina de detecção com $N_{e}=10$ espiras e área de seção transversal de $1 \mathrm{~mm}^{2}$, em um campo magnetostático da ordem de $1 \mathrm{~T}$, o que produz uma magnetização de $3 \mathrm{~mA} / \mathrm{m}$, teremos $\omega_{0} /(2 \pi) \approx 43 \mathrm{MHz}$ e então $\left|V_{\text {ind }}\right|=3,4 \mu \mathrm{V}$, valor que é relativamente simples de detectar e tratar com a eletrônica de RF disponível atualmente. O desenvolvimento de bobinas ortogonais com baixo acoplamento mútuo ou sistemas ressonantes para a detecção de sinal na frequência desejada, trata-se de um grande ramo de estudos em engenharia.

Existem três formas principais para a medida experimental para a NMR, a saber: i) regime de onda contínua (CW - do inglês continuous wave), onde o campo de RF permanece ligado durante toda a medida, e corresponde a uma medida no domínio da frequência, o que requer tipicamente o uso de bobinas ortogonais para excitar e sondar o sistema; ii) regime pulsado, onde são emitidos pulsos cuja duração permite girar a magnetização até o plano, e a partir daí mede-se a tensão induzida produzida pela rotação da magnetização no plano e iii) o regime de eco de spin [22], onde mais de um pulso precisa ser aplicado. Nos dois últimos casos é possível utilizar a mesma bobina para excitar e sondar o sistema, uma vez que as janelas de tempo de aplicação do campo de RF e de medida podem ser diferentes. A análise da operação CW foi feita na Seção anterior deste trabalho. Já a técnica do eco de spin, utilizada em técnicas de alta resolução, não será considerada aqui, por se tratar de uma sequência de pulsos específica que foge ao escopo da presente contribuição. Quanto à técnica de aplicação de pulso, analisando as expressões (34) e (35), sabemos que a magnetização rotaciona completamente para o plano $(x, y)$ após um tempo $\Omega \Delta t=\pi / 2$. É aplicado então um pulso da seguinte forma ao sistema:

$$
b_{x}(t)=b_{0} \cos \left(\omega_{0} t\right)[\Theta(t)-\Theta(t-\Delta t)],
$$

onde $\Theta(\ldots)$ é a função degrau de Heaviside.

A Figura 4 ilustra a dinâmica da magnetização para a configuração de campo pulsado, utilizando a temperatura ambiente e um campo magnetostático de $1 \mathrm{~T}$, o que produz $M_{0} \approx 3 \mathrm{~mA} / \mathrm{m}$, como nos exemplos anteriores. O campo de RF está configurado com amplitude do pulso $b_{0}=1 \mathrm{mT}$, frequência angular $\omega=\omega_{0}$, ou seja, está sintonizado na ressonância, e tem duração $\Delta t=\pi /(2 \Omega)=\pi /\left(\gamma b_{0}\right) \approx 11,74 \mu \mathrm{s}$, por isso também chamado de pulso de $90^{\circ}$. Como se pode observar, a magne-
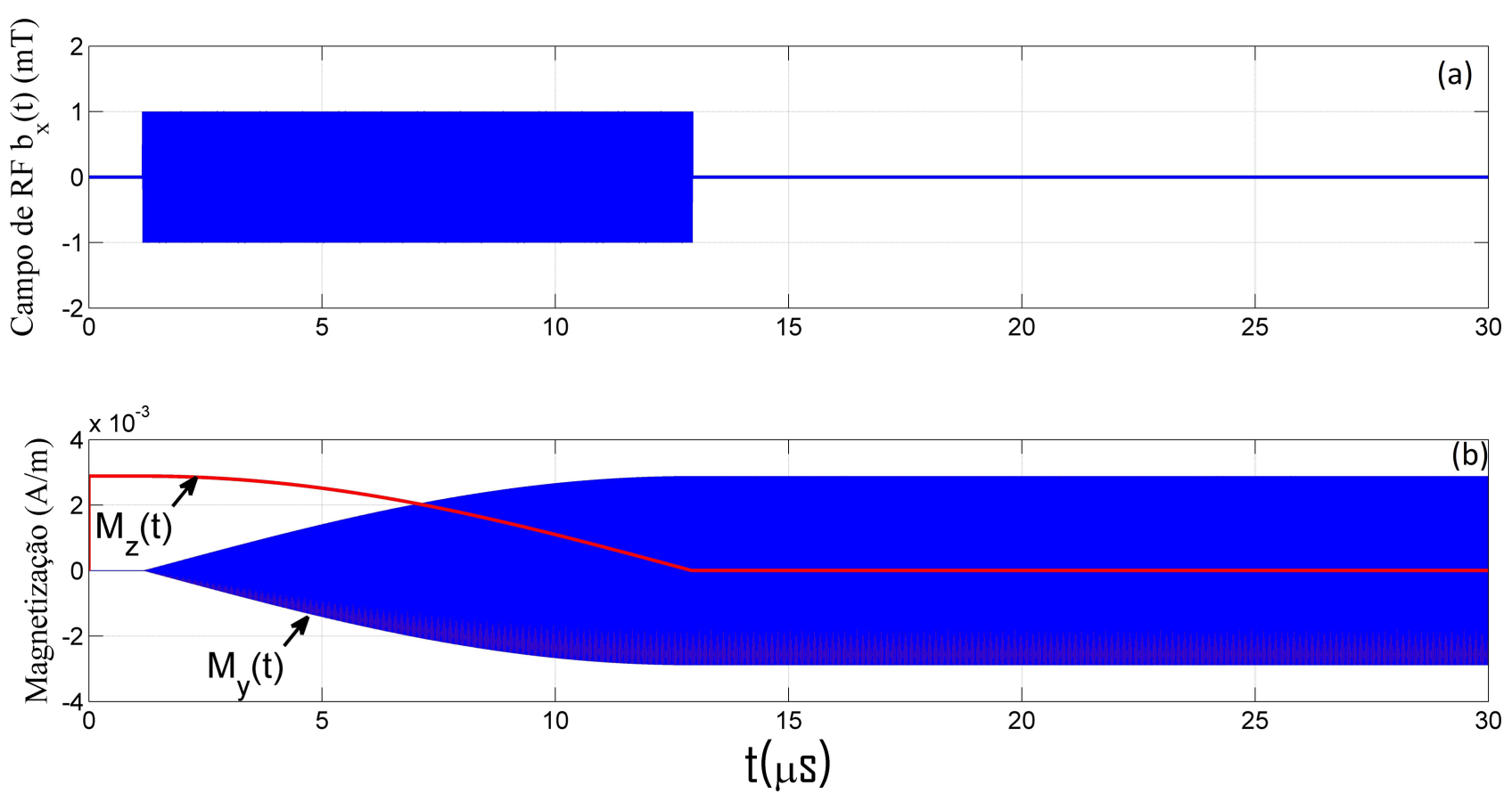

Figura 4: Dinâmica da magnetização na configuração de campo pulsado: (a) Pulso de RF de amplitude $1 \mathrm{mT}$, duração $\pi /(2 \Omega)$ e frequência $\omega=\omega_{0}$ e (b) Magnetização, considerando temperatura ambiente e um campo magnetostático de $1 \mathrm{~T}$. 
tização, que inicialmente está orientada ao longo do eixo $z$, rotaciona para o plano $(x, y)$ e a componente $M_{y}(t)$, que é aquela medida experimentalmente, cresce até o máximo $M_{0}$ e então, uma vez que o pulso de RF cessa, passa a oscilar com amplitude constante e frequência igual à ressonância, devido à precessão da magnetização em torno do eixo z. Na prática, os efeitos de dissipação produzem a atenuação gradual desse sinal, que deve desaparecer, fazendo a magnetização se reorientar ao longo do eixo $z$ em uma escala de tempo da ordem de mili-segundos.

\section{Conclusões}

Neste trabalho procuramos apresentar os conceitos mais fundamentais da ressonância magnética nuclear, obtendo as equações de Bloch a partir do Hamiltoniano de Zeeman para a interação de um momento de dipolo magnético com um campo magnético. Considerações estatísticas sobre a magnetização de um material em alto campo magnetostático foram realizadas e as equações de Bloch foram resolvidas nesse regime de alto campo superposto a um campo magnético de RF, cuja amplitude é tipicamente muito menor do que a do campo magnetostático, porém oscilando com frequência tal que a energia do fóton é suficiente para promover o giro do spin nuclear daquele elemento que se está considerando. Mostrou-se que a variação temporal da componente de magnetização ao longo do eixo do campo magnetostático é lenta, enquanto as componentes transversais rotacionam na frequência de Larmor. Para a NMR as frequências típicas encontram-se na escala de dezenas de megahertz para campos magnetostáticos na escala de $1 \mathrm{~T}$. É importante ressaltar que a NMR é um fenômeno físico melhor descrito pelas leis da mecânica quântica aplicadas ao movimento do spin nuclear. A dinâmica da magnetização proveniente do spin nuclear, por sua vez, produz campos magnéticos variantes no tempo, devido à precessão do próprio spin nuclear, e cuja detecção é relativamente simples, através da utilização de bobinas que medem as tensões induzidas pela rotação da magnetização nuclear. O efeito pode ser observado inclusive em temperatura ambiente e por isso mesmo encontra inúmeras aplicações em Física, Química, Engenharia e Medicina. É utilizada na análise de composição de materiais, tanto sólidos quanto líquidos, e na realização de imagens tomográficas na área médica, sem a necessidade de submeter o paciente à radiação ionizante. Entretanto, as análises dos sinais obtidos em medidas experimentais, para determinar estruturas de proteínas, por exemplo, é tarefa das mais complexa, que envolve pesado processamento de sinais, o que foge ao escopo deste artigo.

\section{Agradecimentos}

C.A. Dartora agradece ao CNPq, pelo suporte financeiro parcial, através de Bolsa de Produtividade em Pesquisa.

\section{Referências}

[1] I.I. Rabi, Phys. Rev. 51, 652 (1937).

[2] I.I. Rabi, J.R. Zacharias, S. Millman and P. Kusch, Phys. Rev. 53, 318 (1938).

[3] J.M.B. Kellogg, I.I. Rabi, N.F. Ramsey and J.R. Zacharias, Phys. Rev. 55, 318 (1939).

[4] E.M. Purcell, H.G. Torrey and R.V. Pound, Phys. Rev. 69, 37 (1946).

[5] F. Bloch, Phys. Rev. 70, 460 (1946).

[6] R.R. Ernst, G. Bodenhausen and A. Wokaun, Principles of NMR in One and Two dimensions (Clarendon Press, Oxford, 1987).

[7] R.R. Ernst, Nuclear Magnetic Resonance Fourier Transform Spectroscopy, Nobel Lecture in Chemistry, 1992.

[8] D.P. Weitekamp, A. Bielecki, D. Zax, K. Zilm and A. Pines, Phys. Rev. Lett. 50, 1807 (1983).

[9] Leo J. Neuringer, Physica B: Condensed Matter 164, 193 (1990).

[10] D.L. Husa and J.G. Daunt, Phys. Lett. A 65, 354 (1978).

[11] Alberto P. Guimarães, Magnetismo e Ressonância Magnética em Sólidos (Edusp, São Paulo, 2009).

[12] A.P. Guimarães, Revista Brasileira de Ensino de Física 22, 360 (2000).

[13] C.P. Slichter, Principles of Magnetic Resonance (Springer, Berlin, 1990), 3rd ed.

[14] K. Wüthrich, Nature Struct. Biol. 8, 923 (2001).

[15] P.C. Lauterbur, Nature 242, 190 (1973).

[16] P. Mansfield and A.A. Maudsley, Brit. J. Radiol. 50, 188 (1977).

[17] P. Mansfield, J. Physics C Solid State Phys. 10, L55-L58 (1977).

[18] J.J. Sakurai, Modern Quantum Mechanics (AddisonWesley, New York, 1994), Revised Ed.

[19] R. M. White, Quantum Theory of Magnetism (Springer, Berlin, 2007), 3rd. Ed.

[20] J.D. Jackson, Classical Electrodynamics (John Wiley \& Sons, New York, 1998), 3rd ed.

[21] Wu, Ying; Yang, Xiaoxue, Phys. Rev. Lett. 98 (2007), 013601.

[22] E.L. Hahn, Physical Review 80 (1950), 580-594. 


\section{Errata: Os fundamentos quânticos da Ressonância Magnética Nuclear}

Erratum: Quantum mechanical principles of Nuclear Magnetic Resonance

No artigo "Os fundamentos quânticos da Ressonância Magnética Nuclear", com número de DOI: http://dx. doi.org/10.1590/1806-9126-RBEF-2017-0093, publicado no periódico Revista Brasileira de Ensino de Física, vol. 40, no 1, e1310 (2018), na Seção 3, página e1310-5 onde se lia:

"Agora podemos impor a condição inicial sobre $M_{z}(t)$, pois sabemos que $M_{z}(0)=M_{0}$, cujo valor é induzido pelo campo magnetostático, conforme discutido na Seção anterior. Essa condição implica que $A_{z}^{+}+A_{z}^{-}=M_{0}$. Admitindo-se que $A_{z}^{+}=A_{z}^{-}=M_{0} / 2$ obtemos uma solução automaticamente real para $M_{z}(t)$. Fica como exercício para o leitor demonstrar que, considerandose a parte real de $m_{y}(t) e^{i \omega_{0} t}$ e na condição inicial apresentada, a solução completa do sistema de equações tem a forma abaixo:

$$
\begin{aligned}
M_{y}(t)= & \frac{M_{0}}{\gamma b_{0}}\left[\Delta \omega \cos (\Omega t) \sin \left[\left(\omega_{0}+\frac{\Delta \omega}{2}\right) t\right]\right. \\
& \left.-2 \Omega \sin (\Omega t) \cos \left[\left(\omega_{0}+\frac{\Delta \omega}{2}\right) t\right]\right], \\
M_{z}(t)= & M_{0} \cos \left(\frac{\Delta \omega}{2} t\right) \cos (\Omega t),
\end{aligned}
$$

onde $\Omega=\frac{1}{2} \sqrt{(\Delta \omega)^{2}+\left(\gamma b_{0}\right)^{2}}$ está relacionada à precessão da magnetização em torno do campo de RF. A determinação da componente $M_{x}(t)$ é feita integrando-se a equação (21), dada a função para $M_{y}(t)$ acima."

\section{leia-se:}

"Agora precisamos impor as condições iniciais sobre as componentes da magnetização. É natural supor que $M_{z}(0)=M_{0}$, cujo valor é induzido pelo campo magnetostático antes da aplicação do campo de RF, conforme discutido na Seção anterior. Ao mesmo tempo é necessário supor a condição inicial sobre as componentes transversais, que naturalmente nos levam a $m_{x}(0)=m_{y}(0)=0$, para poder encontrar os valores de
$A_{z}^{ \pm}$e $A_{y}^{ \pm}$. Fazendo uso da condição inicial $m_{y}(0)=0$, $M_{z}(0)=M_{0}$ e da equação (29) obtemos um sistema para $A_{z}^{+}, A_{z}^{-}$,

$$
\begin{gathered}
\frac{1}{\omega_{y}^{+}} A_{z}^{+}+\frac{1}{\omega_{y}^{-}} A_{z}^{-}=0, \\
A_{z}^{+}+A_{z}^{-}=M_{0}
\end{gathered}
$$

cuja solução é a seguinte:

$$
\begin{gathered}
A_{z}^{+}=\frac{\omega_{y}^{+}}{\omega_{y}^{+}-\omega_{y}^{-}} M_{0}, \\
A_{z}^{-}=-\frac{\omega_{y}^{-}}{\omega_{y}^{+}-\omega_{y}^{-}} M_{0} .
\end{gathered}
$$

Substituindo os valores de $A_{z}^{ \pm}$e $A_{y}^{ \pm}$nas equações (30) e (31), utilizando as expressões para $\omega_{y}^{ \pm}$e $\omega_{z}^{ \pm}$e tomando a parte real, obtém-se o resultado desejado:

$$
\begin{aligned}
M_{y}(t)= & \frac{\gamma b_{0} M_{0}}{2 \Omega} \cos \left[\left(\omega_{0}+\frac{\Delta \omega}{2}\right) t\right] \sin (\Omega t), \\
M_{z}(t)= & M_{0}\left[\cos \left(\frac{\Delta \omega}{2} t\right) \cos (\Omega t)\right. \\
& \left.+\frac{\Delta \omega}{2 \Omega} \sin \left(\frac{\Delta \omega}{2} t\right) \sin (\Omega t)\right]
\end{aligned}
$$

onde $\Omega=\frac{1}{2} \sqrt{(\Delta \omega)^{2}+\left(\gamma b_{0}\right)^{2}}$ está relacionada à precessão da magnetização em torno do campo de RF. A determinação da componente $M_{x}(t)$ é feita integrando-se a equação (21), dada a função para $M_{y}(t)$ acima. É importante lembrar ao leitor que $M_{y}(t)=$ $\operatorname{Re}\left[m_{y}(t) e^{i \omega_{0} t}\right] "$

Cabe destacar que as inconsistências e as devidas correções a este trabalho foram apontadas pelo Prof. Dr. J. Ricardo de Souza, do Departamento de Física, Universidade Federal do Amazonas (UFAM) e também atuante no INCT - National Institute of Science and Technology for Complex Systems, na UFAM, ao qual os autores são imensamente gratos. 